\title{
The clinical utility of routine urinalysis in pregnancy: a prospective study
}

\author{
Noreen Murray, Caroline S E Homer, Gregory K Davis, Julie Curtis, George Mangos and Mark A Brown
}

DURING PREGNANCY many women in Australia routinely have urinalysis. However, there appears to be little evidence to support this practice. ${ }^{1,2}$ There is understandable concern about ceasing urinalysis in pregnant women without evidence for the benefit or safety of this change, particularly for those who may develop proteinuria before hypertension. Thus, an important question is: can urine testing in pregnancy be eliminated without delaying the diagnosis of pre-eclampsia?

We determined whether routine urinalysis during pregnancy should be continued in women with normal results of both urinalysis and microscopic examination of urine at their first antenatal visit.

\section{METHODS}

\section{Study sample}

We used a prospective observational study design. From March to November 1999, all pregnant women attending a hospital antenatal care unit were asked to participate in the study at their initial antenatal visit, and the study was explained verbally and in writing before their written consent was obtained. Nine hundred women from the public hospital antenatal service and 100 women from a nearby private hospital were enrolled in the study. Eighty-seven women were lost to follow-up (miscarriage, relocation or withdrawal from the study for personal reasons), leaving 913 women in the study sample.

\section{ABSTRACT}

Objectives: To determine whether routine urinalysis in the antenatal period facilitates diagnosis of pre-eclampsia. Can routine urinalysis during pregnancy be discontinued in women with normal results of dipstick urinalysis and microscopy at the first antenatal visit?

Design: Prospective observational study.

Setting: A metropolitan public hospital and a private hospital in Sydney (NSW).

Participants: One thousand women were enrolled at their first antenatal visit (March to November 1999), and 913 completed the study.

Outcome measures: The primary outcome was a diagnosis of de novo hypertension (gestational hypertension, pre-eclampsia, or pre-eclampsia superimposed on chronic hypertension).

Results: Thirty-five women had dipstick proteinuria at their first antenatal visit. In $25(71 \%)$ of these women, further dipstick proteinuria was detected during pregnancy, and two (6\%) were diagnosed with pre-eclampsia. Of the 867 without dipstick proteinuria at the first visit, $338(39 \%)$ had dipstick proteinuria $(>1+)$ at some time during pregnancy. There were no statistically significant differences in the proportion of women with and without dipstick proteinuria at their first visit who developed hypertension during pregnancy. Only six women developed proteinuria before the onset of hypertension. Women who had an abnormal result of a midstream urine test at their first visit, compared with women with a normal result, were more likely to have a urinary tract infection diagnosed during pregnancy; however, the numbers were small.

Conclusion: In the absence of hypertension, routine urinalysis during pregnancy is a poor predictor of pre-eclampsia. Therefore, after an initial screening urinalysis, routine urinalysis could be eliminated from antenatal care without adverse outcomes for women.

MJA 2002; 177: 477-480

\section{Procedure for urinalysis}

At the first antenatal visit, a urine sample was collected for dipstick urinalysis, microscopic examination and culture. Women were given written and oral instructions on the collection of a mid-

\section{For editorial comment, see page 468}

St George Hospital, University of New South Wales, and University of Technology, Sydney, Kogarah, NSW.

Noreen Murray, RM, BN, Midwifery Educator, Division of Women's and Children's Health; Caroline S E Homer, RM, PhD, Midwifery Consultant, Conjoint Senior Lecturer, Midwifery Practice and Research Centre; Gregory K Davis, MD, FRACOG, Staff Specialist, Conjoint Senior Lecturer, Division of Women's and Children's Health; Julie Curtis, RN, RM, Clinical Midwifery Specialist, Division of Women's and Children's Health; George Mangos, MD, FRACP, Staff Specialist, Conjoint Senior Lecturer, Department of Medicine and Renal Medicine; Mark A Brown, MD, FRACP, Senior Staff Specialist, Professor of Medicine, Department of Medicine and Renal Medicine.

Reprints will not be available from the authors. Correspondence: Dr Caroline S E Homer, Ward 1W St George Hospital, Gray Street, Kogarah, NSW 2217. homerc@sesahs.nsw.gov.au stream specimen of urine, including perineal cleansing and avoiding skin contact with the collection container. Urine was collected at each subsequent visit using the same procedure.

To perform the dipstick urinalysis, we used Multistix 8SG test strips (Bayer Diagnostics, Victoria, Australia) and an automated urinalysis device (Clinitek 50, Bayer). If proteinuria was detected ( $\geqslant 1+$ protein), the specimen was sent to the laboratory for a spot protein/creatinine ratio $^{3}$ and, if a urinary tract infection was suspected (based on either clinical symptoms or the presence of nitrites and leukocytes on dipstick urinalysis), microscopic examination and culture were performed. 


\section{1: Flow chart of urinalysis detected during pregnancy, and outcomes}

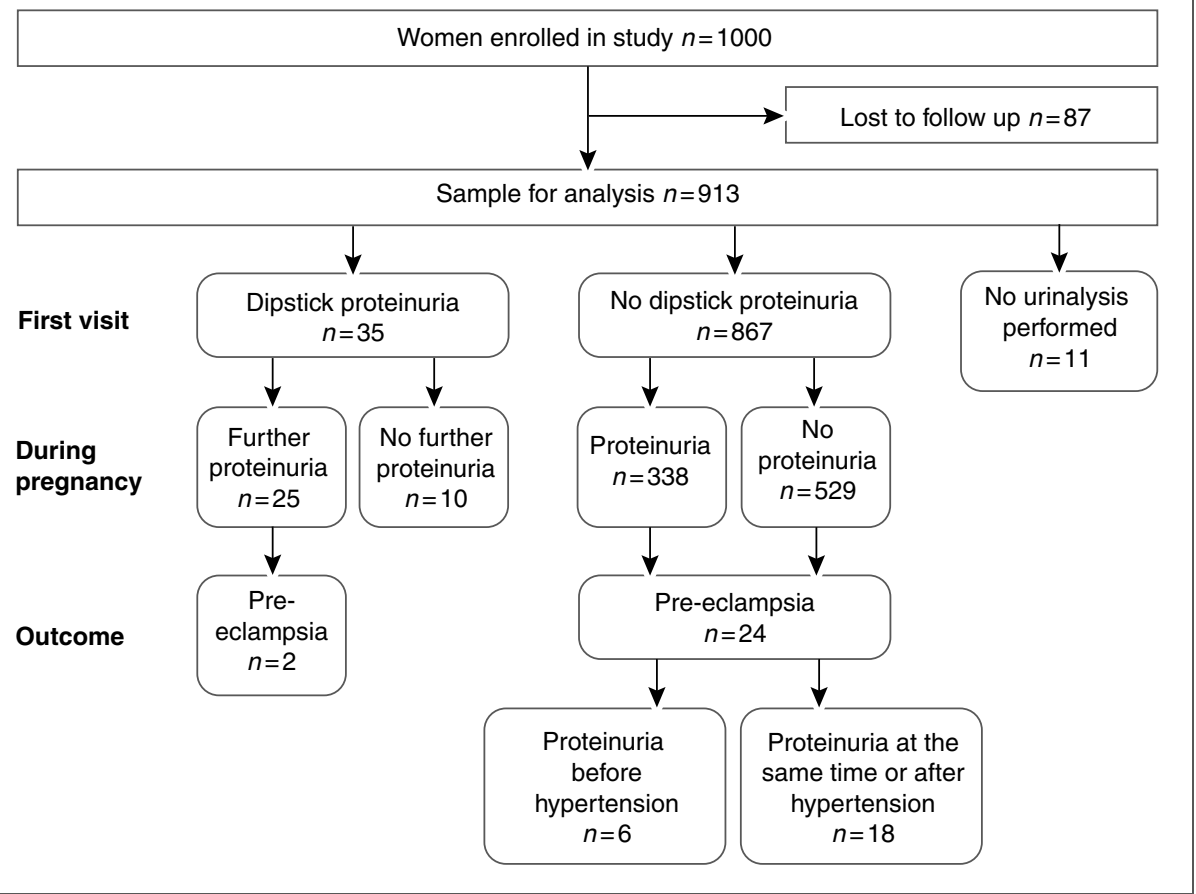

\section{Screening for gestational diabetes}

At the time of the study, all women in our unit were screened for gestational diabetes either at their first visit or at 28 week' gestation using a $50 \mathrm{~g}$ oral glucose challenge test. Women were screened early if they had a history of gestational diabetes, a strong family history of diabetes or glycosuria at the first visit.

\section{Definitions}

- Dipstick proteinuria was defined as $\geqslant 1+$ protein on the automated device. - A midstream urine sample was classified as "normal" if it contained $<10 \times 10^{6} / \mathrm{L}$ erythrocytes, < $10 \times 10^{6} /$ $\mathrm{L}$ polymorphs, and it was culture negative; and as "abnormal" if it contained a pathogen and/or haematuria (erythrocytes $\left.>10 \times 10^{6} / \mathrm{L}\right)$.

- A urinary tract infection was diagnosed when $>10^{6} / \mathrm{L}$ organisms were cultured, with associated pyuria in the absence of epithelial cells.

- Blood pressure measurements (using mercury sphygmomanometry) were taken in accordance with the consensus statement of the Australasian Society for the Study of Hypertension. ${ }^{4}$ - Hypertensive disorders of pregnancy. The definitions used were those of the Australasian Society for the Study

\section{Data collection}

Demographic details, medical and obstetric history were recorded for each woman at the first antenatal visit. The results of blood pressure measurement, dipstick urinalysis and urine microscopic examination and culture were recorded. At each subsequent visit, blood pressure and dipstick urinalysis results were recorded.

The primary outcome was a diagnosis of de novo hypertension (ie, gestational hypertension, pre-eclampsia or preeclampsia superimposed on chronic hypertension).

\section{Ethical approval}

The study was approved by the Institutional Ethics Committee of the South Eastern Sydney Area Health Service (Southern Section).

\section{Statistical analysis}

We determined the relationship between first-visit dipstick proteinuria, pregnancy outcomes and development of Hypertension in Pregnancy. ${ }^{5}$ of hypertension during pregnancy. Clinical outcomes of women who had "normal" versus "abnormal" urine at the first visit and the outcomes of those who subsequently developed proteinuria were compared using $\chi^{2}$ testing. Fisher's exact test was used when the number of observations was less than five.

\section{RESULIS}

The mean age of the 913 women was 29 years (range, 14-48 years) and $48 \%$ $(n=442)$ were nulliparous. Most $(62 \%)$ were from English-speaking backgrounds. Nineteen per cent were from Asian countries and 9\% from Arabicspeaking countries. The remaining 10\% came from European and South American countries. This cultural diversity is representative of the population in the southern suburbs of Sydney where the study was conducted. There were no differences in the demographic characteristics of women in the study sample compared with those who were lost to follow-up (data not shown).

Six per cent $(n=52)$ of women in the study reported previous renal disease, including recurrent urinary tract infection or pyelonephritis $(n=28)$, renal calculi $(n=15)$ and haematuria $(n=5)$, and $2 \%(n=18)$ had had essential hypertension. Of the 471 multiparous women, 11 per cent $(n=50)$ reported previous pre-eclampsia or gestational hypertension.

The flow chart (Box 1) summarises the results of urinalysis in study participants at the first antenatal visit and during pregnancy, as well as the outcomes.

\section{Urine tests at the first antenatal visit and subsequent hypertension}

Eleven women did not have dipstick urinalysis performed at their first visit, although all 913 women had a urine sample collected for microscopic examination and culture.

Thirty-five women had dipstick proteinuria at their first visit, but only 14 had a specimen sent for a spot protein/ creatinine ratio. Of these 14 , two had true proteinuria ( $\geqslant 30 \mathrm{mg}$ protein $/ \mathrm{mmol}$ creatinine). Four of the 35 women with first-visit dipstick proteinuria had a his- 


\section{2: Development of hypertension and pre-eclampsia}

\section{A: According to the result of dipstick urinalysis at the first antenatal visit $(n=902)^{*}$}

\begin{tabular}{lcccc} 
& No proteinuria & Proteinuria $^{\dagger}$ & $\mathbf{P}^{\ddagger}$ & $\begin{array}{c}\text { Relative risk } \\
\mathbf{( 9 5 \%} \mathbf{~ C l})\end{array}$ \\
\hline No hypertension & $\boldsymbol{n}=\mathbf{8 6 7}$ & $\boldsymbol{n}=\mathbf{3 5}$ & & \\
Pre-eclampsia & $809(93 \%)$ & $31(89 \%)$ & 0.2 & $1.8(0.6-4.8)$ \\
Hypertension, other & $24(3 \%)$ & $2(6 \%)$ & &
\end{tabular}

B: In women with no proteinuria at the first antenatal visit $(n=867)$ who subsequently developed dipstick proteinuria during pregnancy

\begin{tabular}{lcccc} 
& $\boldsymbol{n}=\mathbf{5 2 9}$ & $\boldsymbol{n}=\mathbf{3 3 8}$ & & \\
No hypertension & $503(95 \%)$ & $306(91 \%)$ & 0.01 & $1.5(1.1-1.9)$ \\
Pre-eclampsia & $9(2 \%)$ & $15(4 \%)$ & & \\
Hypertension, other & $17(3 \%)$ & $16(5 \%)$ & & \\
\hline
\end{tabular}

*Eleven women did not have dipstick urinalysis performed at their first visit. †Protein $>1+$ on dipstick urinalysis. $\ddagger \chi^{2}$ test for differences between groups. §Includes gestational hypertension, chronic hypertension, and pre-eclampsia superimposed on chronic hypertension.

\section{3: Development of hypertension and subsequent urinary tract infection according to the results of midstream urine tests at the first antenatal visit $(n=913)$}

\begin{tabular}{lcccc} 
& $\begin{array}{c}\text { “Normal” urine } \\
(\boldsymbol{n}=\mathbf{8 3 3})\end{array}$ & $\begin{array}{c}\text { “Abnormal” } \\
\text { urine }^{\star}(\boldsymbol{n = 8 0})\end{array}$ & $\boldsymbol{P}^{\boldsymbol{\dagger}}$ & $\begin{array}{c}\text { Relative risk } \\
\mathbf{( 9 5 \%} \mathbf{~ C l})\end{array}$ \\
\hline No hypertension & $778(93 \%)$ & $73(91 \%)$ & 0.5 & $1.3(0.6-2.8)$ \\
Hypertension & & & & \\
$\quad$ Pre-eclampsia & $22(3 \%)$ & $4(5 \%)$ & & \\
Gestational hypertension & $21(2 \%)$ & $2(2 \%)$ & & \\
Chronic hypertension & $5(1 \%)$ & $1(1 \%$ & & \\
Pre-eclampsia superimposed on & $7(1 \%)$ & 0 & & \\
$\quad$ chronic hypertension & & & & \\
\hline Urinary tract infection during pregnancy & $7(1 \%)$ & $3(4 \%)$ & 0.05 & $4.5(1.2-17)$
\end{tabular}

*Infection and/or haematuria. $+\chi^{2}$ tests for differences between groups; where number of observations $<5$, Fisher's exact test was used.

tory of renal disease. Further dipstick proteinuria was detected during pregnancy in 25 of these 35 women (71\%) and two $(6 \%)$ were diagnosed with preeclampsia.

Of the 867 women without first-visit dipstick proteinuria, $338(39 \%)$ had dipstick proteinuria $(\geqslant 1+)$ at some time during pregnancy. There were no statistically significant differences in the proportion of women with and without first-visit dipstick proteinuria at their first visit who developed hypertension during pregnancy (Box 2A).

\section{Proteinuria during pregnancy and pre-eclampsia}

Of the 338 women (39\%) who developed dipstick proteinuria during preg- nancy, most $(n=325 ; 96 \%)$ had $1+$ proteinuria, with 11 having $2+$, and two women $3+$ proteinuria. Fifteen of the 338 women with dipstick proteinuria (4\%) developed pre-eclampsia, compared with nine of the 529 women $(2 \%)$ without dipstick proteinuria during pregnancy (Box 2B).

Thus, 24 women, who did not have proteinuria at their first visit, developed pre-eclampsia: six had proteinuria diagnosed before the development of hypertension, six had proteinuria identified at the same time as hypertension was diagnosed, and the remaining 12 women developed proteinuria after the diagnosis of hypertension (Box 1).

Of the six women who developed proteinuria (and ultimately pre-eclampsia) before the onset of hypertension, two had multiple pregnancies, one had a history of pre-eclampsia, but none had a history of renal disease. Five of the women delivered at more than 36 weeks' gestation, with the remaining woman delivering at 32 weeks' gestation. There were no adverse neonatal outcomes.

\section{Microscopic examination and culture of urine}

All 913 women had a first-visit midstream urine sample sent to the laboratory for microscopic examination and culture: $91 \%(n=833)$ had a "normal" result and $8 \%(n=80)$ an "abnormal" result (Box 3). Most of the abnormal results $(97 \%)$ were related to the presence of red or white blood cells or sterile pyuria.

Women who had an abnormal midstream urine sample at their first visit were more likely to have a urinary tract infection diagnosed during pregnancy compared with those with a normal midstream urine sample; however, the numbers were small. Four per cent of women $(n=3)$ with an abnormal midstream urine sample developed a urinary tract infection, compared with $1 \%$ of women $(n=7)$ with a normal midstream urine sample $(P<0.05$; relative risk, 4.5; 95\% CI, 1.2-17).

\section{DISCUSSION}

Our results show that routine urinalysis during pregnancy is a poor predictor of the development of pre-eclampsia. We suggest that, after the initial screening urinalysis, routine urine testing could be eliminated from antenatal care in lowrisk women. Only six women in our study had proteinuria diagnosed before the development of hypertension, and no adverse outcomes occurred in this group. Of these six women, three could be described as having risk-associated pregnancies (multiple pregnancy, previous history of pre-eclampsia). Therefore, even if routine urinalysis during pregnancy was not part of antenatal care for low-risk women, these three women would have continued to have urine tests during their pregnancies. In practice, clinical intervention is not instigated until hypertension is 
detected. The detection of proteinuria may mean more intense clinical supervision; however, it is unlikely that cessation of routine urinalysis would have jeopardised the outcomes in these six women.

From previous research on routine urinalysis in pregnancy, it was concluded that, in low-risk women with no objective signs of a hypertensive disorder, routine dipstick proteinuria screening at each antenatal visit does not provide clinically important information about pregnancy outcome. ${ }^{1}$ This research, while useful, was limited by including only women who had no evidence of a hypertensive disorder (including elevated blood pressure, marked oedema and excessive weight gain) during pregnancy. Recent Australasian consensus guidelines do not include the last two clinical features as diagnostic or predictive of hypertensive disorders of pregnancy. ${ }^{5}$ Gribble et $\mathrm{al}^{1}$ also excluded women with pre-existing renal disease or chronic hypertension, multiple pregnancy or proteinuria on the initial urine test. We chose to include all women within the study period to ensure that the study sample was representative of women seen in routine antenatal care.

In clinical practice, routine urinalysis in pregnancy is considered as being useful in the diagnosis of gestational or pre-existing diabetes. At the time of our study, all women in our unit were screened for gestational diabetes using an oral glucose challenge test. Currently, our protocol for screening for diabetes is in line with recommendations of the Australasian Diabetes in Pregnancy Society. ${ }^{6}$ If routine urinalysis during pregnancy were to be omitted, these women would still be identified, either because of glycosuria at the first visit or a positive glucose screening test.

It has also been suggested that urinalysis in pregnancy assists in the detection of asymptomatic bacteriuria. ${ }^{7}$ We would suggest that all women who have "abnormal" results of an initial dipstick urine test should have a midstream urine sample sent for microscopic examination and culture. This would ensure that asymptomatic bacteriuria or underlying renal disease in pregnant women would be detected at their first visit.
While our study protocol mandated that all women with $\geqslant 1+$ proteinuria on dipstick testing should have the sample sent to the laboratory for a spot protein/ creatinine ratio, this did not always occur. This is regrettable, as we know from our previous research that this ratio is a valuable tool in diagnosis of true proteinuria. ${ }^{3}$ Clearly, better education of clinicians in the antenatal services is necessary to ensure that, when dipstick urine testing is done, appropriate follow-up of abnormal results is performed.

Clearly, randomised controlled trials are the ideal study type to address questions of clinical practice such as those posed in our study. However, as this was not feasible, we conducted a prospective observational study. The sample size was selected on feasibility and practicality grounds, and thus there is a potential for a type II error (reporting no difference when indeed there may have been a difference). We acknowledge this limitation, but suggest that, in the circumstances, where units all over Australia have different protocols for urine testing in pregnancy, our study (which provides Level 3 evidence) may provide some clinical guidance for healthcare providers. A further limitation of our study is that no adjustments were made for possible confounders. This was not performed, as the numbers of women with pre-eclampsia were too small.

\section{CONCLUSIONS}

- All low-risk women should provide a correctly collected midstream urine sample at their first antenatal visit for an automated dipstick urine test.

- Women with normal results of an initial dipstick urine test require no further urine tests in pregnancy, unless they develop hypertension or clinical signs and/or symptoms of urinary tract infection.

- Women with abnormal results of a dipstick urine test (including the presence of leukocytes, nitrites or blood) should have a midstream urine sample sent for microscopic examination, culture and sensitivity testing. If the result is asymptomatic bacteriuria, this should be treated appropriately. If the results are normal, no further urine samples are necessary, unless the woman develops hypertension or has symptoms and/ or signs of a urinary tract infection.

- Women found to have true proteinuria and/or haematuria at their first antenatal visit may have underlying renal disease, which should be investigated.

- Routine urinalysis should continue for women with "at-risk" pregnancies (eg, early increase in blood pressure level, essential hypertension, renal disease), as the detection of pre-eclampsia is important in this high-risk group. More research is needed to determine whether routine urinalysis is of value in these women.

\section{COMPEING INTERESTS}

None identified.

\section{ACKNOWLEDGEMENTS}

We would like to thank the midwives who were involved in the recruitment of women and the routine urine testing. We also thank Teresa Baldassarre for her assistance with data entry.

This study was primarily funded by the Midwifery Practice and Research Centre, a National Health and Medical Research Council Centre of Excellence in Hospital-based Research.

\section{REFERENCES}

1. Gribble RK, Fee SC, Berg RL. The value of routine urine dipstick for protein at each prenatal visit. $A m J$ Obstet Gynecol 1995; 173: 214-217

2. Hooper DE. Detecting gestational diabetes and preeclampsia. Effectiveness of routine screening for glucose and protein. J Reprod Med 1996; 41(12): 885-888.

3. Saudan PJ, Brown MA, Farrell TJ, Shaw L. Improved methods of assessing proteinuria in hypertensive pregnancy. $\mathrm{Br} J$ Obstet Gynecol 1997; 104: 1159-1164

4. Australasian Society for the Study of Hypertension in Pregnancy. Management of hypertension in pregnancy: consensus statement. Med J Aust 1993; 158: 700-702.

5. Brown MA, Hague WM, Higgins J, et al. The detection, investigation and management of hypertension in pregnancy. Aust N Z J Obstet Gynaecol 2000; 40: 133-155.

6. Hoffman L, Nolan C, Wilson JD, et al. Gestational diabetes mellitus - management guidelines. The Australasian Diabetes in Pregnancy Society. Med $J$ Aust 1998; 169: 93-97.

7. Smaill F. Antibiotics for asymptomatic bacteriuria in pregnancy (Cochrane Review). The Cochrane Library. Oxford: Update Software, 2002.

(Received 29 Nov 2001, accepted 26 Jun 2002) 